\title{
Potencialização da Educação a Distância com Sistemas Hipermídia Adaptativos: uma Proposta Baseada em Tipos de Personalidade Acadêmica e Recomendação de Conteúdo
}

\author{
Adelito Borba Farias ${ }^{1}$, Lucas Sampaio Leite ${ }^{2}$, Tancicleide Carina Gomes ${ }^{2}$, \\ Bruno Nascimento Figueiredo ${ }^{3}$, Mariana Pereira Xavier ${ }^{1}$ \\ ${ }^{1}$ Centro de Estudos e Sistemas Avançados do Recife (CESAR) \\ Rua Bione, Cais do Apolo - Recife - Brasil \\ ${ }^{2}$ Universidade Federal Rural de Pernambuco (UFRPE) \\ Rua Manuel de Medeiros - Dois Irmãos - Recife - PE - Brasil \\ ${ }^{3}$ Universidade Federal de Pernambuco (UFPE) \\ Av. Jornalista Anibal Fernandes - Cidade Universitária - Recife - Brasil
}

\begin{abstract}
The various features and situations that comprise the formation of a student necessitates customization in content presentation in educational systems to assist in the learning process. In this aspect, this research exposes an optimistic analysis on the adaptation of computing activities through a navigation rule, containing the decisions which must be taken according the level of performance, as well as consider the psychological profile and content recommendations through tags.
\end{abstract}

Resumo. As diversas características e situações que compõem a formação de um estudante torna necessária uma customização na apresentação de conteúdo em sistemas de educação visando auxiliar no processo de aprendizado. Nesta vertente, esta pesquisa expõe uma análise otimista para adaptação de atividades da área de Computação por meio de uma regra de navegação, contendo as decisões que devem ser tomadas a partir do nivel de desempenho, bem como considerar os perfis psicológicos e recomendações de conteúdos por meio de tags.

\section{Introdução}

É um tema recorrente na literatura nacional e internacional a complexidade dos conceitos computacionais que os aprendizes possuem ao iniciarem seus estudos [Piva Jr and Freitas 2010, Mota et al. 2008, Mattos and Fuchs 2007]. Além disso, grande parcela das soluções pedagógicas projetadas para e-learning não analisam as características particulares dos estudantes e o mesmo conteúdo e estratégia pedagógica são comumente usadas para todos que o usam Moodle ${ }^{1}$, Amadeus ${ }^{2}$ e Redu ${ }^{3}$. Este conjunto de fatores pode impactar na vida acadêmica dos estudantes, refletindo, na maior parte dos casos, em desencorajamento, no qual eles optam entre desistir e migrar para outro curso,

\footnotetext{
${ }^{1}$ https://moodle.org

${ }^{2} \mathrm{http}: / /$ amadeus.cin.ufpe.br

${ }^{3}$ http://openredu.cin.ufpe.br/
} 
gerando a evasão; ou trilham o penoso caminho das repetências, perdendo a periodização, atrasando a conclusão do curso.

As pesquisas analisadas identificam alguns fatores que influenciam no insucesso dos estudantes no aprendizado dos conceitos computacionais. Meira e da Silva (2009) detectaram que por meio do estudo da personalidade dos indivíduos, é possível compreender que determinada atividade não se enquadra com os estilos de preferência dele. Esta informação é relevante por auxiliar no bom desempenho de suas atribuições, mitigando as chances de problemas no andamento das atividades. Nesta perspectiva, Paixão et al. (2012) iniciaram estudos nos quais discutem indícios da existência de relação entre o tipo de personalidade e a tendência para um estudante permanecer motivado com as metodologias no ensino de computação.

Nos últimos anos, nota-se aumento na literatura de mecanismos personalizados que viabilizam a escolha de caminhos dentro de um ambiente virtualizado, disponibilizado pelo professor, para facilitar da aprendizagem [Takikawa 2010, Dorça et al. 2011]. Posto isso, Dorça et al. (2011) refletem que as características essenciais do estudante que um ambiente virtualizado devem possuir para contemplar adaptabilidade são: os objetivos de aprendizagem, o nível de conhecimento, os interesses, as preferências, os estereótipos, as preferências cognitivas e os estilos de aprendizagem.

Diversas pesquisas apontam que o ensino quando suportado por medidas instrucionais adequadas aos estilos de aprendizagem dos estudantes, há evoluções no processo de aquisição do saber. Contudo, poucas ferramentas virtuais de ensino fazem uso de estilos de aprendizagem, ou mapeamento cognitivo, no processo educacional [Scaico 2013]. A partir dessa premissa, surgiram os seguintes questionamentos: Será que os exercícios poderiam se adaptar ao perfil, e conhecimentos do estudante? E caso o estudante esteja em erros recorrentes, quais os indicativos para apontar que ele está apto para dar continuidade nos conteúdos, e/ou quais aspectos dos conhecimentos ele precisa melhorar para compreender o conteúdo atual?

Machado et al. (2011) apontam a importância da utilização de técnicas que amparem nossos objetivos a cerca da direção que o aluno deve seguir, caso ele não obtenha bons resultados, diante das tentativas de solucionar atividades de um determinado conteúdo. Diante desta realidade, acredita-se que este trabalho segue uma tendência da atualidade de personalização de conteúdo de forma automatizada e com base no perfil do aluno, e por assim, a presente investigação agrega conhecimento à comunidade científica, por este ter o intuito de viabilizar a inserção de uma regra de navegação que pode ser inserida em diversos modelos de LMS's (Learning Management System) existentes. Para alcançar tal objetivo, a inserção de estilos de aprendizagem se dá com base no indicador MBTI (Myers-Briggs Type Indicator).

Para melhor compreensão de nossa proposta, subdividimos este trabalho nas seguintes seções: a Seção 2 apresenta uma revisão bibliográfica necessária para o entendimento e fundamentação do leitor acerca do tema abordado; a Seção 3 traz uma abordagem explicativa da organização da arquitetura da Camada Pedagógica; por fim, na Seção 4 são apresentadas as considerações e propostas para trabalhos posteriores. 


\section{Hipermídia Adaptativa e Perfis Psicológicos}

A literatura de Inteligência Artificial Aplicada à Educação (IAEd) indica os Sistemas de Tutores Inteligentes (STI) como candidatos fortes para atuar no processo de aprendizagem, por serem capazes de adequar-se ao estilo de aprendizagem de cada estudante a partir de um modelo histórico e do desempenho do estudante. Pimentel et al. (2003) ainda afirma que o processo de auxiliar a aprendizagem não é trivial, requerendo a implementação de sistemas complexos, dinâmicos e adaptativos, sendo o STI adequado para tal atividade.

A organização da arquitetura de um STI varia na literatura científica. Entretanto, segundo Giraffa (1999), elas são descritas seguindo o padrão mínimo de quatro modelos (componentes funcionais), os quais são ditos tradicionais e adotados na maior parte dos STIs desenvolvidos, a saber: Domínio (responsável por representar como as informações são estruturadas e ligadas), Aluno (descreve que tipos de informações, características sobre o aluno devem ser mantidas, tais como: nível de conhecimento, objetivos, histórias e preferências), Tutor/Pedagógico (contêm as estratégias, as táticas de ensino, e é responsável por gerenciar as interações com o aluno) e Interface (viabilizar a comunicação entre aluno e sistema, organizando o diálogo/apresentação das informações).

Palazzo (2000) escreve um Sistema Hipertexto ( $\mathrm{SH}$ ) como uma base de dados detentora de conexões ativas que permitem ao aluno saltar de um ponto para outro em seu interior, conforme desejado. Nesta perspectiva, o termo hipermídia é um acrônimo que combina os conceitos hipertexto e multimídia, no qual viabiliza a criação, alteração, exclusão, compartilhamento e consulta de informações contidas em diversas mídias e permite o acesso da informação de forma não sequencial. No entanto, o controle da navegação não garante que o estudante irá aprender o conteúdo exposto no sistema.

No universo da Ciência da Computação, a Hipermídia Adaptativa (HA) é definida como um sistema que constrói um modelo, por meio de métodos e técnicas, para cada aluno a partir de seu perfil, metas, necessidades, expectativas, preferências e nível de conhecimento, aplicando-os na adaptação de hiperdocumentos e recursos hipermídia vindos de qualquer fonte (bancos de dados, Internet, serviços etc.) [Palazzo 2000].

Os Sistemas Hipermídias Adaptativos (SHA), baseados em STI e SH, ampliam a capacidade de adequação ao perfil do aluno, visto que seu princípio fundamental é o fato de considerar a heterogeneidade entre as pessoas, aonde estes podem aprender em ritmos diferentes, bem como possuir necessidades diferentes de aprendizagem. Assim, torna-se um forte aliado à eficiência do processo de ensino-aprendizagem.

Nesta perspectiva, o presente trabalho está empenhado em modelar as adaptações da navegação dos exercícios, a fim de que a exibição do mesmo conteúdo seja executada de acordo com o caminhar do estudante no curso e nos seus conhecimentos prévios.

Para avaliar padrões de comportamento e perfis psicológicos, fez-se a adoção do indicador MBTI (Myers-BriggsTypeIndicator), que de acordo com Paixão et al. (2012), é a categoria de teste de personalidade mais aplicado em estudos em Computação. Tais perfis são delineados por meio de quatro macros dimensões bipolares da personalidade, a saber: (i) Extraversion/Introversion (Extrovertidos/Introvertidos), (ii) Sensing/iNtuition (Sensoriais/Intuitivos), (iii) Thinking/Feeling(Racionalistas/Emocionais) e (iv) Judging/Perceiving (Julgadores/Perceptivos). 
MBTI julga que os indivíduos intrinsecamente preferem algumas qualidades ou são mais confortáveis com alguns traços do que outros. O traço da personalidade é encontrado através de uma coleção de quatro letras (E ou I, S ou N, T ou F, J ou P), no qual cada letra representa a extremidade principal na dimensão. A cada quarteto que provém dos dezesseis diferentes tipos de personalidade medidas pelo MBTI pode ser interpretado como um conjunto de padrões que indica como o indivíduo se comporta.

Baseado nos perfis psicológicos delineados por Myers-Briggs, Keirsey (1998) descreveu que temperamento é uma representação de inclinações. Esta descrição compõe quatro tipos de personalidade, que com a composição de duas dimensões do indicador MBTI, implicará no temperamento do indivíduo. Tais temperamentos foram classificados em: Artesãos (SP - Sensorial Perceptivo), Guardiões (SJ - Sensorial Julgador), Idealistas (NF- Intuitivo Sentimental) e Racionais (NT - Intuitivo Pensador) conforme exposto na Figura 1.
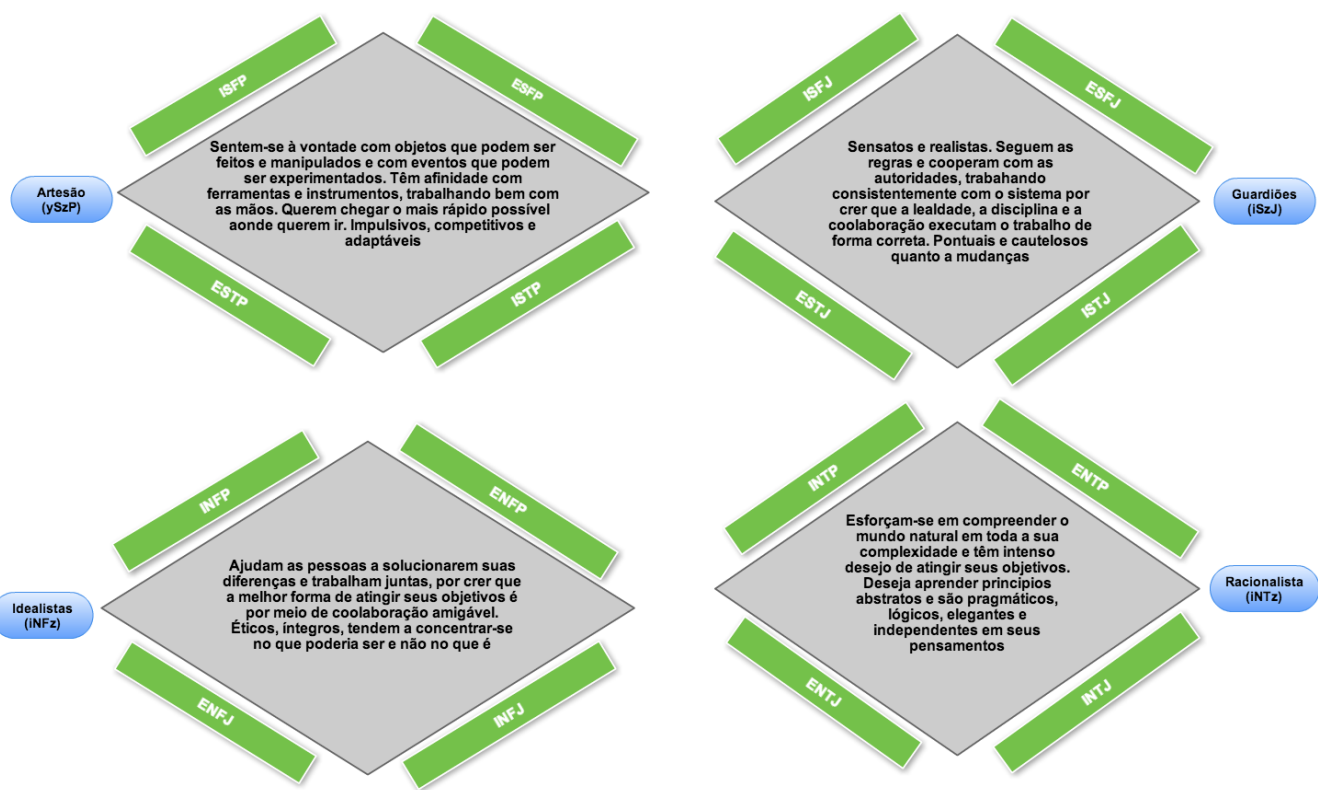

Figura 1. Quatro temperamentos MBTI (adaptado de [Branco et al. 2012])

Após longos períodos estudando o indicador MBTI, Schroeder (2000) notou que o critério de aprendizagem dos alunos era muito mais destacada destacado ao se relacionar com as escolhas de dimensão I/E (Introversão/ Extroversão) e S/N(Sensação/ Intuição). Pesquisas como esta têm coincidido sobre a relação entre o perfil psicológico e as várias perspectivas do processo de formação. Shindler (2005) declarou que essas duas dimensões associadas têm mais aplicação sobre o desempenho acadêmico, onde instituiu "Tipos Acadêmicos" gerando quatro categorias: Extrovertido/Sensitivo, Introvertido/Sensitivo, Extrovertido/Intuitivo e Introvertido/Intuitivo. Estas categorias, mostram que há características que favorece à preferencia de perfis distintos, o que potencializa a adoção deste modelo para esta pesquisa

\section{Arquitetura da Camada Pedagógica}

A literatura investigada apresenta várias pesquisas que abordão a utilização de recursos computacionais para auxiliar o processo de aprendizagem, seja por SHA, por mapeamento 
dos pefis cognitivos, ou por sistema de recomendação (SR). Entretanto, não foi encontrada uma solução educacional que acople estes métodos em prol da facilitação do saber para o aprendente.

Diante deste contexto, pensando em agrupar o que de melhor cada método potencializa, foi projetado um modelo conceitual para ser alinhado com ambientes virtuais de aprendizagem já existente, viabilizando a manutenibilidade arquitetural deste sistemas, e assim, enriquecer os recursos do ponto de vista pedagógico. Neste cenário, a Figura 2 apresenta a arquitetura da Camada Pedagógica, caracterizando-a como um SHA.

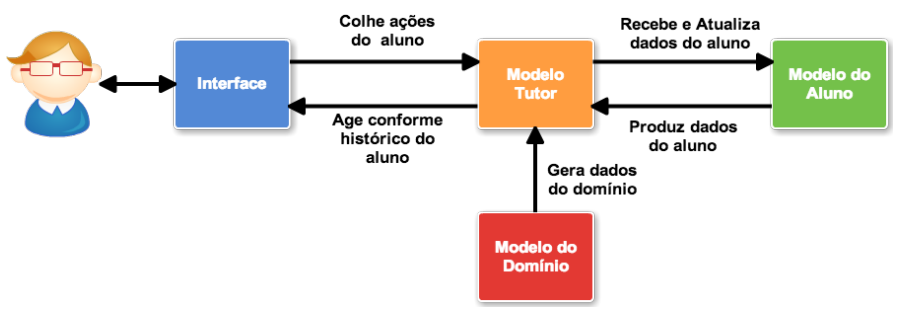

Figura 2. Arquitetura da Camada Pedagógica

No Modelo do Aluno, são quatro conjuntos de dados que o compõe: os registros pessoais como nome de usuário e disciplina matriculada; os registros relativos ao tipo acadêmico; informações sobre o conhecimento prévio do estudante; e os registros sobre o processo de solução do problema, que é descrito na Tabela 1.

Tabela 1. Descrição textual do registro sobre o processo de solução

\begin{tabular}{|c|c|}
\hline Classificação & Descrição Funcional \\
\hline Problema da Seção & Exposição da atividade para o estudante resolver \\
\hline Enunciado & A descrição é exibida de modo que represente uma real situação profissional \\
\hline Explanação & $\begin{array}{c}\text { A ferramenta exibe algumas sugestões que estimule a reflexão para } \\
\text { resolver a atividades selecionadas pelo estudante }\end{array}$ \\
\hline Solução & $\begin{array}{c}\text { A ferramenta expõe a solução da atividade selecionada pelo estudante, } \\
\text { após constatar que não houver êxito na resposta, com o raciocino mais } \\
\text { simples para encontrar a resposta correta para esta atividade }\end{array}$ \\
\hline
\end{tabular}

O Modelo de Aluno proposto é baseado na modelagem do conhecimento sobreposto, visto que se busca conhecer, com o máximo de exatidão, o grau de conhecimento adquirido pelo aluno no decorrer do uso da ferramenta de aprendizagem. Isto se dá a partir de um peso numérico, uma complexidade, que foi conferida a cada atividade para que se possa mensurar o nível de assimilação do conteúdo. Esta complexidade será crucial para o cálculo do rendimento do aluno, atribuído ao Modelo Tutor.

A coleta das informações da interação do aluno no sistema, fonte principal de dados para a atualização do Modelo de Aluno, utilizará formulários/questionários de sondagem, e as respostas fornecidas pelo aluno para cada classe de atividades relacionada a um conteúdo do domínio. O conjunto de dados colhidos será, então, atualizado no Modelo de Aluno pelo questionário inicial preenchido pelo aluno, e a cada vez que acontecer o término de uma atividade executada por ele. Assim, será de conhecimento 
dos estudantes que a ferramenta está o acompanhando em todos os pontos das atividades, coletando as experiências que o estudante está obtendo com a ferramenta e com os conteúdos.

Os problemas propostos aos estudantes deverão receber uma tag associada a uma cor que indica a dificuldade do problema dentro de uma hierarquia que reflete o conhecimento exigido do aprendiz para resolução da questão. A utilização das tags de marcação irá facilitar o modelo do estudante a captar informações relevantes ao aprendizado do aluno associado a um conteúdo da base do domínio, além de expor ao professor quais classes de problemas os estudantes encontram maior dificuldade no momento da resolução.

Os dados do rendimento serão o reflexo da história do aluno na ferramenta, no tocante à assimilação dos conteúdos abordados pela ferramenta educacional. No Modelo Tutor, há regras que direcionam o aluno de acordo com as respostas fornecidas por ele para cada atividade e/ou exercício que será exibido ao longo de seus estudos. Para este direcionamento, tais dados cadastrais serão muito úteis para, diante de alguma dificuldade na resposta de atividades, o sistema, baseado nas notas/rendimentos, pré-requisitos do conteúdo atual que o aluno está trilhando e no seu perfil, sugerir alternativas para melhor compreensão do assunto, que faz parte da funcionalidade do Modelo Tutor.

Para o Modelo de Domínio, que contém o conhecimento que será passado ao estudante, à proposta é a organização da apresentação dos conteúdos educacionais no formato estrutural de livro, com todos os conteúdos subdivididos em capítulos e segmentados em seções, como está descrito na Figura 3, exibindo como se dá a ramificação no livro. Em cada seção, poderá haver mais de uma atividade para mensurar o aprendizado do aluno para aquele conteúdo em questão.

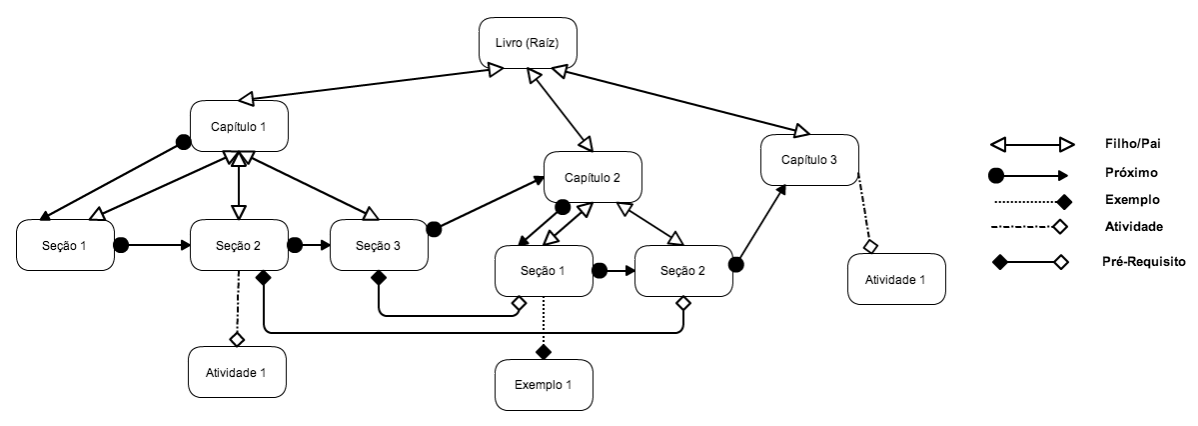

Figura 3. Modelagem do Domínio

Esta estruturação dos conteúdos no Modelo de Domínio tem base na lógica proposta por Garcindo et al. (2002), por possibilitar hierarquização dos conteúdos, prérequisitos para que a regra de navegação possa indicar os conteúdos a serem revisitados caso necessário. Assim, a estruturação se dá a seguir:

- fragmentos: é o nível mais baixo constituído de frações de informação. Então, serão fragmentos parágrafo de texto, imagens, animações;

- nós ou páginas: unidades de apresentação para o aluno. A página é construída à parte e inclui fragmentos de acordo com o mecanismo de adaptação;

- conceitos compostos: o domínio da aplicação pode ser descrito em termos de conceitos de alto nível. A relação entre os conceitos pode existir para indicar 
caminhos de navegação desejáveis. Alguns conceitos fazem parte de um conceito maior numa concepção hierárquica. Pode haver uma relação do tipo um a um entre conceitos e nós (granulação fina), no entanto um conceito poderá envolver uma série de nós.

Há uma sequencia pré-estabelecida para trilhar os conteúdos e que poderão vir com atividades e exemplos. Contudo, caso o estudante tenha alguma dificuldade em algum conteúdo, o sistema irá verificar uma alternativa para auxiliá-lo, e este auxílio poderá ser a reapresentação de conteúdos de maior pré-requisito e que ele tenha obtido baixo rendimento. Por conseguinte a importância da modelagem das seções com os prérequisitos, onde seja possível inserir pesos coerentes com os assuntos que dependem deles, para que a regra de navegação do sistema possa escolher o que tem maior impacto no conteúdo em que o aluno está com dificuldade.

O Modelo Tutor é a chave motriz que guia o processo de adaptação em um SHA. Ele captura as informações do aluno e suas interações no ambiente hipermídia e assegura a atualização do Modelo do Aluno. É nesta perspectiva, que refletimos algumas funcionalidades no Modelo Tutor com a proposta de adaptar as apresentações das atividades das seções através de um conjunto de restrições. Assim, foram projetadas regras específicas para auxiliar o processo de aprendizagem do aluno que irá interagindo com a ferramenta com o objetivo de guiá-lo na obtenção de um rendimento satisfatório para seu aprendizado. A Figura 4 demonstra esquematicamente tais regras.

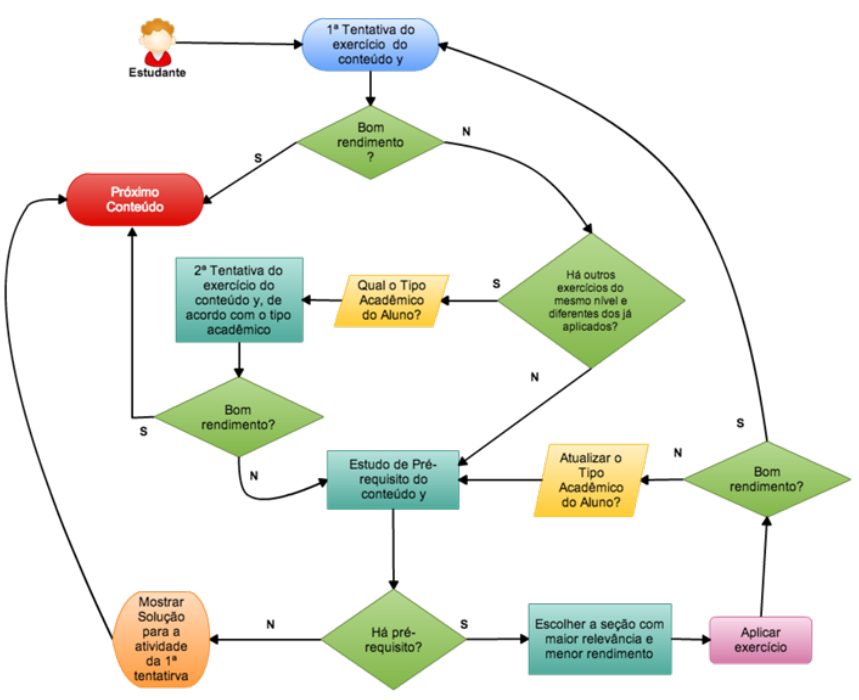

Figura 4. Regras de navegação do Modelo Tutor

Para a definição do rendimento do aluno em cada atividade, foi projetada uma fórmula matemática (Figura 5), que foi arquitetada considerando o fato de que não se deve apenas está preocupado com o que o aluno aprende, mas principalmente, com o que ele deixou de aprender, pois, acreditamos que isto é um potencial risco para o aprendizado efetivo do aluno, existindo a possibilidade dele não ter bom proveito em conteúdos mais complexos ao longo de seus estudos.

A fórmula apresentada na Figura 5, tem por objetivo gerar uma média ponderada entre o nível de complexidade das questões de cada seção. A quantidade de erros 


$$
N_{c a}=\frac{\sum_{i \in T_{c}} d_{c i} \cdot\left(-e_{c i a}\right)}{\sum_{i \in T_{c}} d_{c i}}+1, \forall_{c} \in C, \forall_{a} \in A
$$

Figura 5. Expressão matemática para o cálculo do rendimento

cometidos nas atividades terá valor negativo, justamente pelo fato de acreditar que ele errou por ter deixado de aprender, e assim, um risco para a compreensão dos conteúdos futuros.

Assumem-se os seguintes parâmetros nesta fórmula: $A$ : aluno. $C$ : conjunto dos conteúdos. $T_{c}$ : conjunto de atividades do conteúdo $c . d_{c i}$ : dificuldade da atividade $i$ do conteúdo $c$. $e_{\text {cia }}$ : tentativas do aluno a em realizar a atividade $i$ referente ao conteúdo $c$. $N_{c a}$ : avaliação do aluno $A$ no conteúdo $c$.

Além de ser uma série amplamente conhecida na comunidade científica, e detentora de um arcabouço lógico, que dá credibilidade para ser aplicada em disciplinas de introdução aos conceitos de programação, a sequência de Fibonacci é uma sucessão de números naturais de onde pode ser extraída a Divina Proportione, ou Proporção Áurea. Por ser um número que representa uma constante de crescimento encontrada em vários domínios da natureza, por tal razão, despertou interesse e foi utilizada como base por grandes nomes da humanidade em suas composições intelectuais e artísticas, a exemplo de Leonardo da Vinci (1452-1519), e em teoremas matemáticos recentes, como da busca de Sung et al. (2002), método numérico utilizado para encontrar pontos críticos de funções. Nesta perspectiva, este trabalho adotou a sequência para determinar as complexidades das questões, como está exemplificado na Tabela 2.

Tabela 2. Especificação das complexidades a serem atribuídas às questões

\begin{tabular}{|c|c|}
\hline Complexidade baixa & 3 \\
\hline Complexidade média & 5 \\
\hline Complexidade alta & 8 \\
\hline
\end{tabular}

Após inúmeras simulações, notou-se que os valores obtidos por meio da fórmula tendiam em seguir um padrão numérico, e assim, foi possível estabelecer uma classificação para indicar o rendimento obtido pelo aluno. Desta forma, considerando estas observações, foi estabelecida uma escala para classificar as notas das atividades realizadas pelos alunos, descrita na Tabela 3. Esta classificação também está embasada na sequência de Fibonacci.

Tabela 3. Especificação dos intervalos para atribuição de rendimento

\begin{tabular}{|c|c|}
\hline Intervalo & Atribuição \\
\hline 1 e 0,5 & 8 \\
\hline 0,5 e 0 & 5 \\
\hline 0 e $-0,5$ & 3 \\
\hline$-0,5$ e -1 & 2 \\
\hline-1 em diante & 1 \\
\hline
\end{tabular}


É importante destacar que o padrão de notas adotado neste trabalho não visa atribuir pontuação que desestimulem o estudante nas etapas da construção do saber. Assim, acredita que a nota 1 é o bastante para alertar o aluno que seu desempenho não está satisfatório, e merece maior dedicação com os estudos.

\section{Considerações Finais e Trabalhos Futuros}

Este trabalho apresentou uma proposta que visa contribuir em uma nova arquitetura com funcionalidades pedagógicas adicionais para a Camada Pedagógica para ser inserida em um ambiente virtual de aprendizagem, cujo objetivo é auxiliar no ensino de conceitos computacionais, baseada em interação e visualização. Essa arquitetura inclui uma regra de navegação construída com o objetivo de indicar qual ação deverá ser tomada, partindo do resultado do rendimento do aluno e do perfil detectado pelo indicado MBTI. Para a obtenção do rendimento, uma fórmula foi elaborada para considerar os erros como um risco para a aprendizagem do aluno, e uma classificação para atribuir um valor numérico para o rendimento. Por fim, foi definida uma estrutura de conteúdo para estabelecer as ligações entre as seções dos capítulos do livro eletrônico.

Realizado estudo literário, foi constatada a necessidade de inserir os mecanismos de adaptação por meio de sistemas hipermídia, por facilitar a detecção dos pontos onde os estudantes estão no conteúdo, e por ser viável a agregação de multimídias que auxiliam o potencial pedagógico do ambiente virtual de aprendizagem.

São grandes os desafios de elaborar ferramentas pedagógicas que democratizem e qualifiquem os processos de ensino-aprendizagem. Neste momento da pesquisa, os esforços foram dispendidos para detectar uma solução plausível para os modelos essenciais de um sistema adaptativo - Tutor, Domínio, Estudante e Interface. Diante disso, a arquitetura proposta tem a missão de extrair o perfil do estudante ao interagir com o ambiente de aprendizagem, potencializando o aprendizado, por buscar estratégias para assimilação dos conteúdos, atenuando possíveis evasões escolares.

Uma análise mais cautelosa será realizada para mitigar questionários cansativos e muitas vezes não respondidos corretamente, a fim de obter isenção de interferências provocadas pelo esgotamento mental e físico que prejudiquem o aprendizado. Além disso, a próxima iteração desta pesquisa, haverá participação de professores especialistas no conteúdo da disciplina adotada, a fim de validar de forma empírica a classificação das complexidades dadas às questões, e assim, verificar objetivamente quão impactante a arquitetura se apresenta no processo de aprendizagem.

\section{Referências}

Branco, D., Conte, T., Prikladnicki, R., and Conte, T. (2012). Um estudo preliminar sobre tipos de personalidade em equipes scrum. CIbSE, pages 304-311.

Dorça, F. A., Lima, L. V., Fernandes, M. A., and Lopes, C. R. (2011). Detecção e correção automática de estilos de aprendizagem em sistemas adaptativos para educação. Revista de Informática Teórica e Aplicada, 18(2):178-204.

Garcindo, L. A. S. et al. (2002). Uma abordagem sobre o uso da hipermídia adaptativa em ambientes virtuais de aprendizagem. 195p. Tese (Doutorado)- Programa de Pósgraduação em Engenharia de Produção. Universidade Federal de Santa Catarina. Florianópolis. 
Giraffa, L. M. M. (1999). Uma arquitetura de tutor utilizando estados mentais. 177p. Tese (Doutorado)- Programa de Pós-graduação em Computação. Universidade Federal do Rio Grande do Sul. Porto Alegre.

Keirsey, D. (1998). Please Understand Me II. Prometheus Nemesis Book Company.

Machado, J. B., Silva, M. A., Maldonado, J. C., and Barbosa, E. F. (2011). Reengineering of educational content: an experience in the computer networks domain. XXII Simpósio Brasileiro de Informática na Educação, SBIE(2011). Aracaju/SE., 1(1).

Mattos, M. and Fuchs, J. (2007). Qualifica: Um método automatizado para apoio ao ensino de algoritmos. Revista Eletrônica de Sistemas de Informação da UNIME, 1:2036.

Meira, A. F. and da Silva, F. Q. (2009). Habilities and behavioural profiles of sqa professionals related to process maturity levels. Software Engineering. XXIII Brazilian Symposium on, pages 136-144.

Mota, M. P., Pereira, L. W. K., and Favero, E. L. (2008). Javatool: uma ferramenta para ensino de programação. XXVIII Congresso da Sociedade Brasileira de Computação. Belém/PA., pages 127-136.

Paixão, C., Fortaleza, L. L., and Conte, T. (2012). Um estudo preliminar sobre as implicações de tipos de personalidade no ensino de computação. XXXII Congresso da Sociedade Brasileira de Computação, XX WEI, Curitiba/PR.

Palazzo, L. A. M. (2000). Modelos proativos para hipermídia adaptativa. 114p. Tese (Doutorado)- Programa de Pós-graduação em Computação. Universidade Federal do Rio Grande do Sul. Porto Alegre.

Pimentel, E. P., França, V. F. d., Noronha, R. V., and Omar, N. (2003). Avaliação contínua da aprendizagem, das competências e habilidades em programação de computadores. Anais do Workshop de Informática na Escola, 1(1):533-544.

Piva Jr, D. and Freitas, R. L. (2010). Estratégias para melhorar os processos de abstração na disciplina de algoritmos. XXI Simpósio Brasileiro de Informática na Educação, SBIE(2010). João Pessoa/PB., 1(1).

Scaico, Q. (2013). Ta educação do futuro: uma refexão sobre aprendizagem na era digital. II Congresso Brasileiro de Informática na Educação.

Schroeder, C. C. (2000). New students—new learning styles. Dezure, D.

Shindler, J. (2005). Teaching for the success of all learning styles: Five principles for promoting greater teacher effectiveness and higher student achievement for all students. Los Angeles.

Sung, V. S. H., Silva, K. S., Barros, T. E., and Carbone, V. L. (2012). Sequência de fibonacci e suas aplicações. Monografia (Graduação)- Departamento de Matemática. Universidade Federal de São Carlos. São Carlos.

Takikawa, F. K. (2010). Arquitetura de sistemas hipermídia adaptativos baseada em atributos de qualidade. Dissertação (Mestrado)-Programa de Pós-Graduação em Engenharia da Computação e Sistemas Digitais. Universidade de São Paulo, São Paulo. 